\title{
General behavior of the effective nucleon-nucleon interaction as a function of the relative velocity
}

\author{
L. C. Chamon \\ Instituto de Física da Universidade de São Paulo, Caixa Postal 66318, 05315-970 São Paulo, SP, Brazil \\ M. A. G. Alvarez \\ Departamento de Fisica Atomica Molecular y Nuclear (FAMN), Apartado 1065, Universidade de Sevilla, E-41080, Sevilla, Spain
}

(Received 23 March 2005; published 7 July 2005)

\begin{abstract}
We have studied volume integrals of the central part of optical potentials extracted from data analyses for a variety of light and heavy systems. The data-extracted integrals present a quite simple behavior as a function of the relative velocity between target and projectile. This behavior is system independent and, therefore, it reflects a feature of the effective nucleon-nucleon interaction itself. The overall results are in good agreement with the predictions of the São Paulo potential, which is a model for the nuclear interaction that so far has been employed mostly in analyses of heavy-ion reactions.
\end{abstract}

DOI: 10.1103/PhysRevC.72.014603

PACS number(s): 24.10.Ht, 21.30.Fe, 25.10.+s

\section{INTRODUCTION}

Elastic scattering of nucleus-nucleus systems has been an important subject of study for several decades. Within the context of the optical model, such studies usually have been performed with the aim of determining the corresponding mean-field interaction. Indeed, the optical model has provided good data fits to angular distributions of numerous different systems in a wide energy range. The resulting optical potential parameters should provide physical information on the nuclear interaction. However, in several cases the data-extracted potentials are rather ambiguous in the sense that different sets of parameters result in quite similar data fits. This is clearly observed at near-barrier energies where potentials that are similar in the surface region give equivalent fits to the data. At these low energies, the Coulomb repulsion and strong absorption prevent the projectile from penetrating the target and therefore it probes only the surface region. With the advent of higher energy beams, much less ambiguity has been observed in the extraction of the optical potential at short distances from data analyses at intermediate energies. Such data were first obtained for $\alpha$-particle scattering and later for several heavyion systems [1]. The resulting phenomenological potentials present significant dependence on the bombarding energies, and theoretical models have been developed to account for this energy dependence through realistic mean-field interactions. Among them, the São Paulo (SP) potential associates this behavior with nonlocal quantum effects related to the exchange of nucleons between target and projectile [2-4]. The SP potential has successfully been used in the data description of a large number of heavy-ion systems [3,5-13]. In the present paper, we show that the energy dependence of volume integrals obtained for potentials extracted from data analyses is in agreement with the predictions of the SP potential [4], not only for heavy-ion systems but also for quite light ones, such as nucleon-nucleus and the $\alpha$ nucleus.

\section{SÃO PAULO POTENTIAL}

Pauli nonlocality arises from quantum exchange effects and has been studied in the context of neutron-nucleus [14], $\alpha$ nucleus [15], and heavy-ion collisions [2-13]. The nonlocal interaction has been assumed in the description of the elastic scattering process through an integro-differential equation $[3,4,14]$. It is possible to define a local-equivalent potential that, within the usual framework of the Schrödinger differential equation, reproduces the results of the integro-differential approach $[3,4,14]$. For heavy-ion systems, we proposed a model for the real part of the local-equivalent interaction, the SP potential, which is defined through two equivalent versions [4]. The first version is a conventional folding of the nucleon distributions of the nuclei with an effective nucleon-nucleon interaction

$$
V_{N}(R)=\int \rho_{1}\left(\vec{r}_{1}\right) \rho_{2}\left(\vec{r}_{2}\right) u_{n n}\left(\vec{R}-\vec{r}_{1}+\vec{r}_{2}\right) d \vec{r}_{1} d \vec{r}_{2}
$$

The SP potential consists in using Eq. (1) in the context of the folding-type nucleon-nucleon interaction

$$
u_{n n}(r)=\int \rho_{m}\left(\vec{r}_{1}\right) \rho_{m}\left(\vec{r}_{2}\right) V_{0} \delta\left(\vec{R}-\vec{r}_{1}+\vec{r}_{2}\right) e^{-4 v^{2} / c^{2}} d \vec{r}_{1} d \vec{r}_{2},
$$

where $\rho_{m}$ is the matter density of the nucleon, $V_{0}=$ $-456 \mathrm{MeV} \mathrm{fm}^{3}, v$ is the local relative velocity between the nucleons (or nuclei), and $c$ is the speed of light. Because of the delta function, the folding in Eq. (2) is called the zerorange approach. Based on the results for the charge density of the proton in free space, obtained from electron scattering experiments, we assumed an exponential shape, $\rho_{m}(r)=\rho_{0} e^{-r / a_{m}}$, for the matter density of the nucleon. This assumption results in

$$
u_{n n}(r)=\frac{V_{0}}{64 \pi a_{m}^{3}} e^{-r / a_{m}}\left(1+\frac{r}{a_{m}}+\frac{r^{2}}{3 a_{m}^{2}}\right) e^{-4 v^{2} / c^{2}} .
$$

A diffuseness value of $a_{m}=0.30 \mathrm{fm}$ was found [4] for the matter density of the nucleon inside the nucleus.

The other version of the SP potential is obtained through the matter density $\rho_{M}$ of the nucleus, which is defined as the folding of the nucleon distribution of the nucleus with the 
matter density of the nucleon:

$$
\rho_{M}(r)=\int \rho\left(\overrightarrow{r^{\prime}}\right) \rho_{m}\left(\vec{r}-\vec{r}^{\prime}\right) d \overrightarrow{r^{\prime}}
$$

Thus, we distinguish the matter and nucleon densities of the nucleus by taking into account the finite size of the nucleon. By inserting Eqs. (2) and (4) in Eq. (1), the SP potential can be recast as

$$
V_{N}(R)=\int \rho_{M 1}\left(\vec{r}_{1}\right) \rho_{M 2}\left(\vec{r}_{2}\right) V_{0} \delta\left(\vec{R}-\vec{r}_{1}+\vec{r}_{2}\right) e^{-4 v^{2} / c^{2}} d \vec{r}_{1} d \vec{r}_{2} .
$$

The similar form of Eqs. (5) and (2) implies a quite interesting unification of the nucleus-nucleus and effective nucleonnucleon interactions: Both are calculated by folding the corresponding matter distributions in the zero-range approach with the same $V_{0}$ value. Furthermore, the same dependence on the relative velocity is present in both kinds of interaction. The model also includes systematics for the densities [4]. Within this context, the SP potential does not contain any adjustable parameter and therefore it is a totally parameter free model for the real part of the nuclear interaction.

An essential characteristic of the SP potential is the dependence on the local relative velocity $v(R)$. In a classical physics framework, the velocity is related to the kinetic energy $E_{K}(R)$ by

$$
v^{2}=\frac{2 E_{K}}{\mu}=\frac{2}{\mu}\left[E-V_{C}(R)-V_{N}(R)\right]
$$

where $\mu$ is the reduced mass of the system, $E$ is the energy in the center-of-mass frame and $V_{C}$ is the Coulomb potential. Although Eq. (6) connects energy and velocity, if one takes into account the form of expressions (2) and (5) it seems more appropriate to consider the nuclear interaction as velocity dependent instead of energy dependent.

A standard physical quantity in optical model analyses is the volume integral per nucleon of the real part of the nuclear interaction:

$$
J_{R}=\frac{4 \pi}{A_{1} A_{2}} \int_{0}^{\infty} V_{N}(R) R^{2} d R .
$$

Within the SP model, a simple expression for $J_{R}$ is obtained if, as an approximation, we replace in Eq. (5) the local velocity by the asymptotic one, $v_{\infty}=v(R \rightarrow \infty)$, that is,

$V_{N}(R) \approx V_{0} e^{-4 v_{\infty}^{2} / c^{2}} \int \rho_{M 1}\left(\vec{r}_{1}\right) \rho_{M 2}\left(\vec{r}_{2}\right) \delta\left(\vec{R}-\vec{r}_{1}+\vec{r}_{2}\right) d \vec{r}_{1} d \vec{r}_{2}$.

In this case and taking into account the normalization of the densities, one obtains

$$
J_{R}=V_{0} e^{-4 v_{\infty}^{2} / c^{2}} .
$$

One should observe that Eq. (9) is deduced from Eqs. (7) and (8) without any hypothesis on the shape of the densities. Thus, according to the SP model, the volume integral per nucleon should be approximately a system-independent quantity with a simple dependence on the relative velocity between target and projectile.

\section{VOLUME INTEGRAL SYSTEMATICS}

For heavy-ion systems, usually the optical potential involved in data analyses presents only central real and imaginary parts. For lighter systems, other kind of interactions, such as spin-orbit, are often included in the calculations. In the present work, we consider only the volume integral per nucleon of the central real part of the optical potential. Considering the ambiguities present in the determination of optical potential parameters from data analyses, one should expect similar ambiguities in the corresponding volume integrals. For light systems, in spite of ambiguities found for the data-extracted parameters, the volume integral of the interaction is a rather well-defined quantity, free of parameter correlations (see, e.g., [16]). For heavy systems, the degree of ambiguity is greater at low energies, near the barrier. Thus, we selected potentials extracted from data at higher energies and mostly from analyses involving realistic potentials based on the folding model. Even so, some degree of ambiguity still remains. For instance, for ${ }^{16} \mathrm{O}+{ }^{16} \mathrm{O}$ at $E_{\mathrm{Lab}}=480 \mathrm{MeV}$ it is possible to obtain good elastic scattering data fits within about $40 \mathrm{MeV} \mathrm{fm}^{3}$ uncertainty, that is, with $J_{R}$ ranging from 242 to $279 \mathrm{MeV} \mathrm{fm}^{3}$ $[17,18]$.

Figure 1(a) shows the data-extracted volume integrals [17-23] for a few heavy-ion systems as a function of the asymptotic velocity that was calculated within the scenario of the theory of relativity:

$$
E_{K}=\frac{m_{0} c^{2}}{\sqrt{1-v^{2} / c^{2}}}-m_{0} c^{2}
$$

where $m_{0}$ and $E_{K}$ are the rest-mass and asymptotic kinetic energy of the projectile, respectively. The straight line in Fig. 1(a) represents the SP potential prediction [Eq. (9)]. The system-independent slope of the "data" is clearly compatible with the velocity dependence of the theoretical model. However, the value $V_{0}=-456 \mathrm{MeV} \mathrm{fm}^{3}$ from the SP potential seems to be too large to represent the data. This discrepancy is, in fact, not very significant, because the SP potential has been successfully used in the elastic scattering data description for several heavy-ion systems, also including a few angular distributions that correspond to volume integral data presented in Fig. 1(a). Thus, we believe that such a discrepancy is due to the approximation assumed in the deduction of Eq. (9), of using the asymptotic velocity, and also due to the ambiguity already mentioned in the determination of volume integrals from elastic scattering data analyses. To illustrate this point with an example, Fig. 2 presents an elastic scattering angular distribution for the ${ }^{12} \mathrm{C}+{ }^{12} \mathrm{C}$ system at $E_{\mathrm{Lab}}=1016 \mathrm{MeV}$. The solid line in the figure was obtained from optical model calculations, where the SP potential and a Woods-Saxon potential (with three adjustable parameters) were assumed for the real and imaginary parts of the interaction, respectively. As one can see in Fig. 2, the agreement between data and theoretical results is excellent. In this case, the exact volume integral of the SP potential, obtained from Eq. (7), is $J_{R}=227 \mathrm{MeV} \mathrm{fm}^{3}$, whereas expression (9) results in a slightly greater value $J_{R}=241 \mathrm{MeV} \mathrm{fm}^{3}$. Similar fits of the same angular distribution have also been obtained within other models for the nuclear interaction, with volume integrals that 


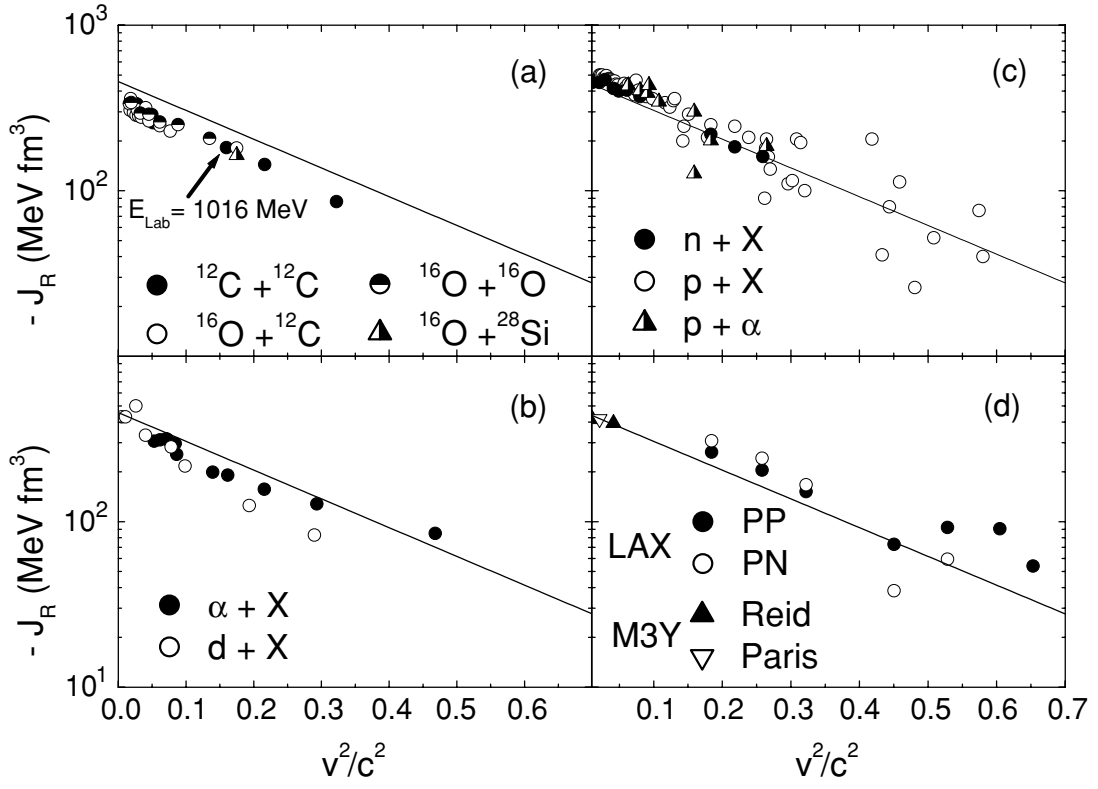

FIG. 1. Volume integrals per nucleon as a function of the asymptotic relative velocity for (a) heavy-ion systems, (b) $\alpha, d+$ nucleus, (c) nucleon + nucleus, and (d) effective nucleonnucleon interactions. The solid lines in the figure represent Eq. (9). The arrow in (a) points to the date that represents earlier results $[17,20]$ obtained from analyses of the angular distribution presented in Fig. 2. vary from 175 to $189 \mathrm{MeV} \mathrm{fm}^{3}$ [17,20] [see arrow in Fig. 1(a)]. The range $175 \leqslant J_{R} \leqslant 227 \mathrm{MeV} \mathrm{fm}^{3}$ of values compatible with this angular distribution shows, again, that a dispersion of $\Delta J_{R} \approx 50 \mathrm{MeV} \mathrm{fm}^{3}$ is expected for $J_{R}$ values extracted from elastic scattering data analyses.

Figure 1(b) shows volume integrals for $\alpha$-nucleus and deuteron-nucleus systems. The data correspond to average values over a large number of different target nuclei [25]. Volume integrals for nucleon-nucleus systems are presented in Fig. 1(c). Again the data correspond to average values over several nuclei [26]. Also in Fig. 1(c), we present data for the very light system proton $+\alpha$ [27].

As shown in Figs. 1(a)-1(c), Eq. (9) is in reasonable agreement with the data for all heavy and light systems studied in the present work. The behavior of the volume integral

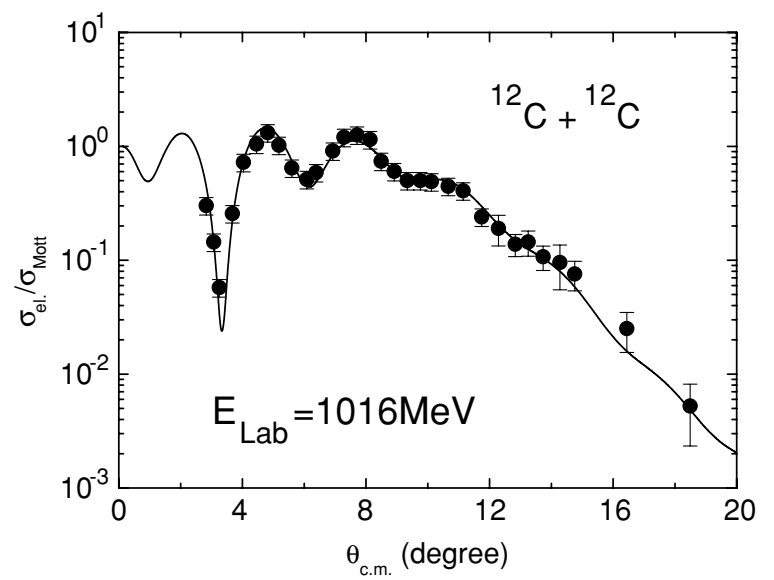

FIG. 2. Elastic scattering angular distribution for the ${ }^{12} \mathrm{C}+{ }^{12} \mathrm{C}$ system at $E_{\mathrm{Lab}}=1016 \mathrm{MeV}$ (from [24]). The solid line represents theoretical results from optical model calculations with the parameterfree SP potential assumed for the real part of the interaction. as a function of the velocity is clearly system independent, and therefore it probably reflects a feature of the effective nucleon-nucleon interaction itself. Obviously, the folding type nucleon-nucleon interaction [Eq. (2) or (3)] obeys Eq. (9). We have checked whether other models for the nucleon-nucleon interaction are also in agreement with that equation. Two different versions, Paris and Reid, of the M3Y interaction have been used to describe low-energy (5-20 MeV/nucleon) elastic scattering data [28]. Another model used in the analyses of elastic scattering is the LAX interaction [29], which is the optical limit of the Glauber high-energy approximation. The LAX interaction is essentially a zero-range double-folding potential used for both the real and imaginary parts of the optical potential. The real part of the LAX interaction is written as

$$
V(R)=-\alpha(E) \frac{\hbar v}{2} \sigma\left(E_{N}\right) \int \rho_{1}(\vec{r}) \rho_{2}(\vec{R}-\vec{r}) d \vec{r},
$$

where $\sigma$ is the energy-dependent total nucleon-nucleon cross section. The parameter $\alpha$ is defined by $\operatorname{Re} f_{n n}=\alpha \operatorname{Im} f_{n n}$, where $f_{n n}$ is the nucleon-nucleon amplitude. The volume integral per nucleon corresponding to Eq. (11) is given by

$$
J_{R}=-\frac{\hbar v}{2} \alpha(E) \sigma(E) .
$$

Equation (11) has been derived from multiple-scattering theories and should be valid only at high energies. In Fig. 1(d) we present volume integrals for the M3Y and LAX effective nucleon-nucleon interactions. For the LAX interaction, we have obtained values for $\sigma$ and $\alpha$ in table 1 of Ref. [29], for both proton-proton $(p p)$ and proton-neutron $(p n)$ interactions. One can see in Fig. 1(d) that the M3Y and LAX interactions present a similar behavior in comparison with that of the folding type nucleon-nucleon interaction.

In Fig. 3 (bottom), we present the same volume integral data set of Fig. 1, but on a linear scale to show that the nuclear interaction becomes repulsive for $v \geqslant 0.7 c$, whereas 


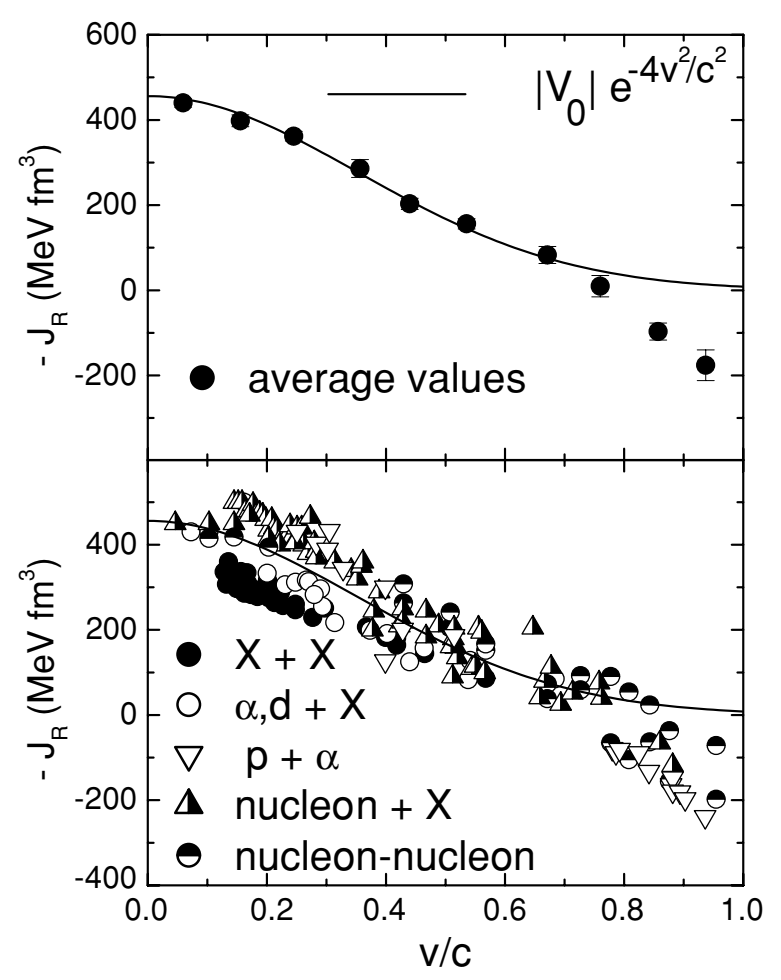

FIG. 3. (Bottom) Volume integrals per nucleon as a function of the asymptotic relative velocity. (Top) Average values of the volume integrals over $0.1 \mathrm{v} / \mathrm{c}$ bins. The solid lines in the figure represent Eq. (9).

the prediction of the SP potential vanishes. However, for $v \leqslant 0.7 c$ the data follow the behavior of Eq. (9). In this region, the standard deviation of the data relative to the theoretical predictions is $\Delta J_{R} \approx 70 \mathrm{MeV} \mathrm{fm}{ }^{3}$. This value is close to the aforementioned data dispersion $\left(40-50 \mathrm{MeV} \mathrm{fm}^{3}\right)$ expected as a result of the ambiguity in the determination of $J_{R}$ from elastic scattering data-analyses. Therefore, within the inherent uncertainty of data-extracted $J_{R}$, the complete data set (for $v \leqslant 0.7 c$ ) is in agreement with the theoretical predictions. To obtain smaller uncertainty, in Fig. 3 (top) we present average values over $0.1 \mathrm{v} / \mathrm{c}$ bins for the complete volume integral data set. The agreement of these average values with the prediction of the SP potential is remarkable up to about $0.7 c$. Indeed, for $v \leqslant 0.7 c$ the dispersion of the average data around the

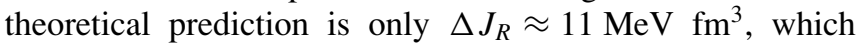
corresponds to about $2.4 \%$ of the $V_{0}$ value. We point out that such very good precision was obtained without using any adjustable parameter in the calculations.

In several works (see, e.g., [25-27]), the volume integrals have been parametrized as a function of the kinetic energy per nucleon $\left(E_{N}\right)$ with the following expression:

$$
J_{R}=J_{0}+\beta \ln E_{N} .
$$

This type of energy dependence is in accord with Passatore's [30-32] application of Feshbach's dispersion relation [33]. Figure 4 presents the average volume integral values as a function of the kinetic energy per nucleon. The solid line in

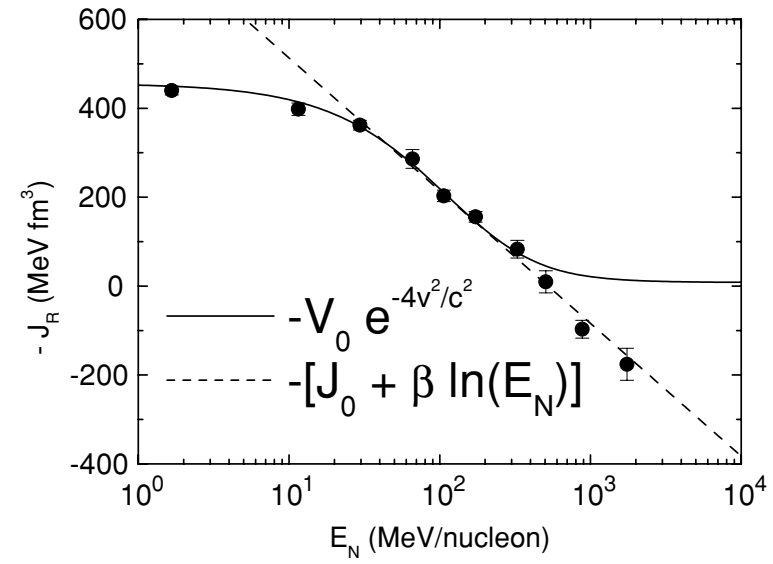

FIG. 4. Average values for the volume integrals as a function of the energy per nucleon. The solid and dashed lines represent Eqs. (9) and (13), respectively.

Fig. 4 represents Eq. (9); the dashed line corresponds to a fit to the data in the region $E_{N}>20 \mathrm{MeV} /$ nucleon using Eq. (13). We found $J_{0}=-812 \mathrm{MeV} \mathrm{fm}^{3}$ and $\beta=130 \mathrm{MeV} \mathrm{fm}^{3}$, which should be compared, for example, with $J_{0}=-872 \pm 44 \mathrm{MeV}$ $\mathrm{fm}^{3}$ and $\beta=136 \pm 7 \mathrm{MeV} \mathrm{fm}^{3}$ obtained for proton-nucleus systems [26]. Figure 4 shows that Eqs. (9) and (13) provide very similar $J_{R}$ values for $30 \leqslant E_{N} \leqslant 300 \mathrm{MeV} /$ nucleon, which corresponds to $0.25 \leqslant v / c \leqslant 0.65$. For low energies Eq. (9) is much better than Eq. (13) whereas the inverse is observed at very high velocities.

\section{CONCLUSION}

We have studied volume integrals of the central real part of optical potentials extracted from elastic scattering data analyses for a large variety of systems. The behavior of the volume integrals per nucleon as a function of the relative velocity between target and projectile is clearly system independent and therefore it reflects a characteristic of the effective nucleon-nucleon interaction itself. The dependence of these integrals on the relative velocity is compatible with the prediction of the SP potential up to $v \approx 0.7 c$. So far, the SP potential had been tested only up to $E=200 \mathrm{MeV} /$ nucleon [3,5], which represents $v \approx 0.57 c$, and it has been employed mostly in the study of heavy-ion reactions. The present findings indicate that the model probably could be successfully applied also in elastic scattering data analyses of much lighter systems.

\section{ACKNOWLEDGMENTS}

This work was partially supported by Fundação de Amparo à Pesquisa do Estado de São Paulo (FAPESP), Conselho Nacional de Desenvolvimento Científico e Tecnológico (CNPq), and the Spanish MEC. 
[1] M. E. Brandan and G. R. Satchler, Phys. Rep. 285, 143 (1997).

[2] M. A. Candido Ribeiro, L. C. Chamon, D. Pereira, M. S. Hussein, and D. Galetti, Phys. Rev. Lett. 78, 3270 (1997).

[3] L. C. Chamon, D. Pereira, M. S. Hussein, M. A. Candido Ribeiro, and D. Galetti, Phys. Rev. Lett. 79, 5218 (1997).

[4] L. C. Chamon, B. V. Carlson, L. R. Gasques, D. Pereira, C. De Conti, M. A. G. Alvarez, M. S. Hussein, M. A. Candido Ribeiro, E. S. Rossi, Jr., and C. P. Silva, Phys. Rev. C 66, 014610 (2002).

[5] L. C. Chamon, D. Pereira, and M. S. Hussein, Phys. Rev. C 58, 576 (1998).

[6] M. A. G. Alvarez, L. C. Chamon, D. Pereira, E. S. Rossi, Jr., C. P. Silva, L. R. Gasques, H. Dias, and M. O. Roos, Nucl. Phys. A656, 187 (1999).

[7] L. R. Gasques, L. C. Chamon, C. P. Silva, D. Pereira, M. A. G. Alvarez, E. S. Rossi, Jr., V. P. Likhachev, B. V. Carlson, and C. De Conti, Phys. Rev. C 65, 044314 (2002).

[8] M. A. G. Alvarez et al., Phys. Rev. C 65, 014602 (2002).

[9] E. S. Rossi, Jr., D. Pereira, L. C. Chamon, M. A. G. Alvarez, L. R. Gasques, J. Lubian, B. V. Carlson, and C. De Conti, Nucl. Phys. A707, 325 (2002).

[10] L. R. Gasques, L. C. Chamon, D. Pereira, M. A. G. Alvarez, E. S. Rossi, Jr., C. P. Silva, G. P. A. Nobre, and B. V. Carlson, Phys. Rev. C 67, 067603 (2003).

[11] L. R. Gasques et al., Phys. Rev. C 67, 024602 (2003).

[12] M. A. G. Alvarez, L. C. Chamon, M. S. Hussein, D. Pereira, L. R. Gasques, E. S. Rossi, Jr., and C. P. Silva, Nucl. Phys. A723, 93 (2003).

[13] L. R. Gasques, L. C. Chamon, D. Pereira, M. A. G. Alvarez, E. S. Rossi, Jr., C. P. Silva, and B. V. Carlson, Phys. Rev. C 69, 034603 (2004).

[14] F. Perey and B. Buck, Nucl. Phys. 32, 353 (1962).

[15] D. F. Jackson and R. C. Johnson, Phys. Lett. B49, 249 (1974).

[16] G. W. Greenlees, W. Makofske, and G. J. Pyle, Phys. Rev. C 1, 1145 (1970).
[17] D. T. Khoa, W. von Oertzen, and H. G. Bohlen, Phys. Rev. C 49, 1652 (1994).

[18] D. T. Khoa, W. von Oertzen, H. G. Bohlen, G. Bartnitzky, H. Clement, Y. Sugiyama, B. Gebauer, A. N. Ostrowski, Th. Wilpert, M. Wilpert, and C. Langner, Phys. Rev. Lett. 74, 34 (1995).

[19] D. T. Khoa and G. R. Satchler, Nucl. Phys. A668, 3 (2000).

[20] M. E. Brandan, Phys. Rev. Lett. 60, 784 (1988).

[21] J. Y. Hostachy, M. Buenerd, J. Chauvin, D. Lebrun, Ph. Martin, J. C. Lugol, L. Papineau, P. Roussel, N. Alamanos, J. Arvieux, and C. Cerruti, Nucl. Phys. A490, 441 (1988).

[22] A. A. Ogloblin, Y. A. Glukhov, V. Trzaska, A. S. Dem'yanova, S. A. Goncharov, R. Julin, S. V. Klebnikov, M. Mutterer, M. V. Rozhkov, V. P. Rudakov, G. P. Tiorin, Dao T. Khoa, and G. R. Satchler, Phys. Rev. C 62, 044601 (2000).

[23] D. T. Khoa, W. von Oertzen, H. G. Bohlen, and F. Nuoffer, Nucl. Phys. A672, 387 (2000).

[24] M. Buenerd, A. Lounis, J. Chauvin, D. Lebrun, P. Martin, G. Duhamel, J. C. Gondrand, and P. De Santignon, Nucl. Phys. A424, 313 (1984).

[25] A. Nadasen, S. Balaji, J. Brace, K. A. G. Rao, P. G. Roos, P. Schwandt, and J. T. Ndefru, Phys. Rev. C 68, 014613 (2003).

[26] A. Nadasen, S. Balaji, J. Brace, K. A. G. Rao, P. G. Roos, P. Schwandt, and J. T. Ndefru, Phys. Rev. C 66, 064605 (2002).

[27] L. G. Arnold, B. C. Clark, and R. L. Mercer, Phys. Rev. C 19, 917 (1979).

[28] G. R. Satchler and W. G. Love, Phys. Rep. 55, 183 (1979).

[29] M. S. Hussein, R. A. Rego, and C. A. Bertulani, Phys. Rep. 201, 279 (1991).

[30] G. Passatore, Nucl. Phys. A95, 694 (1967).

[31] G. Passatore, Nucl. Phys. A110, 91 (1968).

[32] G. Passatore, Nucl. Phys. A248, 509 (1975).

[33] H. Feshbach, Ann. Phys. (NY) 5, 357 (1958). 